Revue de l'Institut des langues et cultures

d'Europe, Amérique, Afrique, Asie et Australie

$35 \mid 2019$

Intermédialités dans les arts et la littérature de l'Espagne $\left(X X^{e}\right.$ et $X X I^{e}$ siècles)

\title{
Daniela Astor y la caja negra, de Marta Sanz : rencontre entre la fiction narrative et le documentaire audiovisuel
}

Daniela Astor y la Caja Negra, by Marta Sanz: When Narrative Fiction Meets Audio-Visual Documentary

\section{Myriam Roche}

\section{(2) OpenEdition Journals}

Édition électronique

URL : http://journals.openedition.org/ilcea/6069

DOI : 10.4000/ilcea.6069

ISSN : 2101-0609

Éditeur

UGA Éditions/Université Grenoble Alpes

Édition imprimée

ISBN : 978-2-37747-083-9

ISSN : 1639-6073

Référence électronique

Myriam Roche, «Daniela Astor y la caja negra, de Marta Sanz : rencontre entre la fiction narrative et le documentaire audiovisuel», ILCEA [En ligne], 35 | 2019, mis en ligne le 27 mars 2019, consulté le 19 mai 2020. URL : http://journals.openedition.org/ilcea/6069 ; DOI : https://doi.org/10.4000/ilcea.6069

Ce document a été généré automatiquement le 19 mai 2020.

(C) ILCEA 


\title{
Daniela Astor y la caja negra, de Marta Sanz : rencontre entre la fiction narrative et le documentaire audiovisuel
}

\author{
Daniela Astor y la Caja Negra, by Marta Sanz: When Narrative Fiction Meets \\ Audio-Visual Documentary
}

Myriam Roche

1 Le roman de Marta Sanz, Daniela Astor y la caja negra, paru en 2013 aux éditions Anagrama, suit les premiers pas dans l'adolescence d'une petite fille de douze ans, qui raconte à la première personne sa vie quotidienne et les évènements qui vont la bouleverser dans le contexte de la transition démocratique espagnole. Le texte, d'une extrême subtilité dans l'expression de la vie intérieure de la jeune protagoniste, fait alterner le récit narratif principal avec ce qui semble être le script d'un documentaire audiovisuel, proposant ainsi une modalité littéraire d'intermédialité très peu courante, voire inédite à cette échelle. Même si la nature de l'œuvre demeure intégralement textuelle ${ }^{1}$, il existe bien une forme d'hybridation médiatique dans la mesure où l'on constate «la présence au sein d'un artefact donné de formes relevant, au départ, de médias différents » (Besson, 2014) : une simple « coprésence », selon la typologie établie par le chercheur Rémy Besson, suffisante pour déclencher de très riches effets de sens chez les auteurs qui souhaitent l'exploiter.

\section{Le documentaire fictif}

2 L'alternance des deux fils narratifs surprend le lecteur dès la page 17, sans aucune forme de transition apparente, en venant interrompre un récit qui a débuté quelques pages plus tôt. La typographie accompagne le changement par l'utilisation de caractères gras, puis de parenthèses qui encadrent le premier paragraphe de texte sans 
que cet effet de ponctuation prenne immédiatement sens pour le lecteur. Les premiers éléments d'ouverture du documentaire audiovisuel sont un titre mis en évidence par l'italique ( La caja negra »), puis une indication de la nature du genre ( Un documental $»)$, le nom de l'auteure-réalisatrice, une année en chiffres romains, et enfin une dédicace : autant de procédés de présentation qui peuvent relever aussi bien d'une œuvre littéraire que d'un film documentaire, ce qui souligne d'entrée la possibilité d'une parenté entre les deux médias. Le lien avec le récit principal est établi par l'identité de la réalisatrice, "Catalina $H$. Griñán", un nom que le lecteur peut immédiatement associer à celui de la jeune narratrice qui a ouvert le roman par ces mots : " Me llamo Catalina Hernández Griñán. Tengo doce años » (Sanz, 2013 : 11). Certaines clés de compréhension du roman sont ainsi révélées : d'une part le décalage temporel entre les deux âges, fondamental dans la confrontation des regards portés sur une période et sa culture populaire; d'autre part l'effacement partiel du nom du père, que le lecteur ne pourra interpréter qu'à la lecture des évènements racontés dans la suite de l'intrigue.

3 Le premier mot pour introduire le générique de début du film, avant même la première des dix parties du documentaire (la «Caja 1 »), est «Imágenes», comme pour mieux baliser le terrain de l'intermédialité: celle-ci passe par une forme particulière d'ekphrasis, à mi-chemin entre le synopsis préparatoire au tournage et la description pure de ce que le (télé)spectateur a devant les yeux lors de son visionnage. L'intérêt porté aux aspects techniques, tout comme l'utilisation du vocabulaire et du style qui leur sont associés, appartient a priori à un document de type professionnel, mais certaines marques de subjectivité, dans le regard ou dans la voix, esquissent l'existence d'une instance narratrice incarnée et impliquée, que la suite de notre analyse essaiera de démasquer. La présence de plusieurs médias est soulignée par les descriptions de procédés cinématographiques qui jalonnent cette introduction : les premières images sont télévisées, mais d'autres, issues d'internet, apparaissent rapidement en surimpression; puis un travelling arrière dévoile l'appareil de télévision, lui-même installé sur la scène d'un café-concert ; enfin, c'est par un fondu au noir que se termine l'ouverture du documentaire avant de laisser place à sa première partie. Cette façon d'associer deux sources d'image, puis d'inclure leur contenant dans un contexte théâtral, invite à considérer la possibilité d'une mise en abyme intermédiale, à plusieurs dimensions de surcroît puisqu'au-delà de cette configuration structurelle il existe des liens thématiques de même nature entre les deux niveaux de récit: le documentaire porte sur des actrices célèbres grâce au cinéma ou au petit écran, et pour lesquelles Catalina, la jeune narratrice, éprouve une fascination immense, au point de vouloir leur ressembler, de chercher à les imiter, d'interpréter leurs rôles en se dédoublant par l'imaginaire.

Le même type d'effet de sens est activé par les titres : celui du roman inclut celui du documentaire par la mention d'une " caja negra », une boîte noire polysémique qui peut désigner tout autant l'objet télévision (boîte à images), que l'enregistreur de vol des avions, qui conserve paramètres de voyage et conversations des pilotes. Le rapprochement entre les deux sens est naturel, en lien avec les thèmes du roman, dans la mesure où la télévision est le vecteur d'une certaine forme de mémoire collective et populaire. Les productions audiovisuelles, bien que destinées par nature à une diffusion unique et éphémère, sont archivées par des institutions ${ }^{2}$ et ont même droit aujourd'hui à de multiples secondes vies sur internet; elles constituent de la sorte un fonds 
documentaire de leur époque, comme c'est le cas dans Daniela Astor y la caja negra : tout en déroulant son intrigue, le roman dresse en arrière-plan le portrait d'une époque complexe, celle de la transition démocratique. Chacune des parties du documentaire est appelée «caja », porte un numéro, et consiste en un fragment relativement autonome, consacré à un élément d'analyse précis : une actrice ${ }^{3}$, un phénomène culturel, un film. Jusqu'au dernier chapitre, acmé paroxystique de la manipulation et de l'exploitation (ici consenties) de toutes ces femmes, vedettes de bas étage transformées en femmesobjets et livrées en pâture aux téléspectateurs.

5 La «Caja 10 », intitulée "Los platós bárbaros ", nous transporte à une époque plus contemporaine que les précédentes et cible clairement la télévision, ou plutôt une certaine catégorie de productions audiovisuelles : «Imágenes de un programa del corazón que una cadena privada emite en horario de máxima audiencia» (Sanz, 2013: 243). Le présentateur vedette y met en scène, avec la complicité de l'actrice invitée, une sorte de tribunal des temps modernes ("los platós bárbaros parecen salas donde se imparte la justicia », [Sanz, 2013: 248]) pour contraindre son interlocutrice à faire la révélation publique d'une relation homosexuelle passée, sous les applaudissements déchainés du public. Tout semble écrit d'avance, comme dans une pièce de théâtre où les acteurs récitent leurs répliques, et celles-ci sont d'ailleurs introduites selon le code typographique propre aux versions écrites des œuvres théâtrales : un retour à la ligne suivi d'un espace blanc, puis le nom du personnage en majuscules et deux points pour introduire le texte. L'analogie est renforcée par la phrase "Comienza el espectáculo" (Sanz, $2013: 244$ ) placée juste avant le début du dialogue, et vient rappeler la mise en abyme initiale du documentaire : le poste de télévision posé sur une scène de théâtre populaire. Autant de signes intermédiaux permettant de souligner, à l'ère du simulacre, le caractère préfabriqué de ce type d'émissions qui reposent entièrement sur l'illusion et la manipulation. Le constat traverse les époques puisqu'il vaut aussi bien pour le reality show des années 2000 que pour les actrices vedettes du destape auxquelles s'intéresse le film. Bárbara Rey, invitée de l'émission mise en scène dans ce dernier chapitre du livre, incarne par son propre parcours la façon pathétique dont certaines n'ont fait que passer d'un leurre à l'autre.

Le choix d'associer au roman la catégorie du documentaire est tout à fait essentiel dans le questionnement du rapport entre réalité et fiction, qui imprègne tous les niveaux de l'intrigue. Selon la définition du documentariste Didier Mauro, « le documentaire est un cinéma faisant création du réel» (Mauro, 2005: 28), ce qui implique la notion de restitution directe du réel, associée à une démarche artistique, et lui confère un statut de document, voire de témoignage selon les cas, sur une époque donnée. Le roman, dont la nature est par essence fictionnelle, accueille donc en son sein non seulement un autre média mais aussi une autre modalité de rapport au réel, et devient par là-même hybride. Cela lui permet d'apporter un double éclairage sur un phénomène culturel, le destape, et ses actrices.

7 Le premier point de vue est celui de Catalina à 12 ans : elle admire et fantasme les égéries de l'époque, qu'elle considère comme des modèles de féminité, reproduit leurs attitudes ou leurs paroles, rêve d'une vie d'adulte semblable à celle qu'elle leur imagine. Caractérisée par le manque de discernement ou d'esprit critique, cette attitude est assez typique de l'âge de la protagoniste, à mi-chemin entre l'enfance et la véritable adolescence, en quête de repères pour la construction d'une identité sexuée et sexuelle. Fascinée par des images dans lesquelles elle se projette, elle demeure ignorante de la 
réalité qui se cache derrière. Alors, comme en contrepoint, le rôle du documentaire est non seulement d'informer sur le devenir de ces femmes-objets mais aussi de dévoiler ce que le simulacre dissimule, de proposer une traversée du miroir, ou de l'écran, pour donner accès au réel. En d'autres termes, le documentaire fait œuvre de démythification, à destination des lecteurs du roman tout comme des spectateurs des émissions ou des films cités, et les condamne à une certaine forme de trouble ou de malaise.

8 Le processus psychologique est très similaire à l'évolution du personnage de la jeune Catalina, qui peu à peu abandonne le monde du fantasme (celui de son double imaginaire Daniela Astor), pour mieux s'ancrer dans la dure réalité des évènements tragiques qui touchent sa famille; elle découvre ainsi la lucidité comme un mal nécessaire, inévitablement à l'origine d'une forme d'angoisse. Dans l'extrait suivant, cette angoisse est paradoxalement exprimée à l'aide de références cinématographiques, ce qui semble souligner que la réalité et l'imaginaire s'entremêlent encore chez la jeune fille à ce stade de son évolution :

La realidad me pega a la tierra y Daniela Astor se me escapa. Me digo que no importa. Que tengo que verlo todo con los ojos abiertos. Aunque el aire no me llegue a los pulmones y sepa que voy a ver imágenes que no podré olvidar. Como la cabeza de la niña de El exorcista o las garras de Lorelei. (Sanz, 2013 : 131)

9 L'épreuve est si difficile que Catalina cherchera encore à lui échapper plus tard, lorsque sa mère sera emprisonnée pendant de longs mois et que le travail scolaire sera son seul exutoire :

Durante este curso saco las mejores notas de toda mi vida. Pero eso no es bueno. Es malo. Esas notas son una mierda. El síntoma de un dolor. Porque me meto en los libros como en una sepultura. Para que no me llegue la luz. Para olvidar la verdad. (Sanz, 2013 : 215)

10 Fuir la lumière et la vérité : la tentation est grande de détourner le regard, de rester dans le monde du strass et des paillettes, mais le documentaire vient justement nous en empêcher, nous lecteurs aussi bien que Catalina, future documentariste engagée, qui porte déjà en elle l'adulte qu'elle sera: "Ésta es una historia sobre el adulto que todos los niños llevamos dentro y también sobre la niña que se ha quedado dentro de mí» (Sanz, 2013 : 173).

\section{Points de vue et interactions}

11 La proximité du documentaire avec la démarche journalistique, essentiellement dans son rapport au réel, pourrait laisser croire que les deux points de vue sont semblables, mais il n'en est rien. Les documentaristes assument depuis longtemps un rejet de l'objectivité chère à la plupart des journalistes, s'inscrivant ainsi dans une intention plus artistique que scientifique, et radicalement subjective :

Une analyse prédomine: tout film est explicitement ou implicitement orienté (consciemment ou inconsciemment). Ceci, parce que l'auteur, le réalisateur, sont porteurs d'un vécu, d'une culture, d'une affectivité, de tensions pulsionnelles, d'un regard sur le monde, et que dès lors que l'on tourne une caméra vers une direction déterminée, ce dispositif est déjà un choix, une orientation. (Mauro, 2005 : 35-36)

12 Ce constat, valable pour toute œuvre cinématographique, amène dans le cas particulier $\mathrm{du}$ documentaire à poser la question de l'engagement: traiter un sujet souvent d'actualité avec un point de vue subjectif correspond nécessairement à une prise de position, qui en théorie pourrait n'être qu'esthétique mais dans les faits prend très 
souvent une dimension éthique voire franchement politique. Dans le roman de Marta Sanz, la documentariste, c'est-à-dire Catalina adulte, fait effectivement des choix très marqués dès la conception même de son œuvre, qu'il s'agisse des thèmes mis en avant ou de leur organisation. Chacune des parties introduit un sujet d'analyse qui vient illustrer le propos de la réalisatrice sur les femmes, avec une forme de progression qui peut faire sens. La caja 1 s'ouvre ainsi :

(Voz en off sobre foto fija: la cámara se mueve sobre la foto analizada deteniéndose en detalles al hilo del off. Los detalles fotográficos se transforman en dibujos coloreados en el momento en que la cámara se centra en ellos. La persona se hace personaje y la realidad se convierte en representación). (Sanz, $2013: 18)$

Le documentaire audiovisuel, lui-même intégré dans le roman, invite à l'ekphrasis d'une photographie, même si le commentaire en voix off est plus analytique que descriptif: l'intermédialité passe donc par une nouvelle forme de mise en abyme ou d'enchâssement, juste après celle de l'introduction, ce qui fait de ce procédé un motif récurrent du roman, essentiel pour tisser le lien entre les différents médias. La photo décrite est celle de l'actrice Susana Estrada, dont la veste trop échancrée découvrit un sein nu lors d'une cérémonie de remise de prix par le futur maire de Madrid, Enrique Tierno Galván, en février 1978. Ce cliché, repris dans toute la presse nationale et même mondiale, est devenu emblématique de l'époque de la Transition et fait partie de la mémoire collective de tous les espagnols. Il fait office de propos liminaire au documentaire, et permet au spectateur d'entrer de plain-pied à la fois dans la période concernée, en activant ses propres souvenirs, et dans la thématique soulevée, celle du destape, avec toute son ambiguïté. Le sein de Susana Estrada, symbole de la nouvelle liberté acquise après quarante ans de dictature et étrennée avec une insolente légèreté, est aussi l'étape initiale d'un processus de marchandisation du corps féminin qui s'instaure dans un premier temps avec la complicité souriante de ses actrices, ignorantes sans doute des effets pervers déplorables que le phénomène aura sur la perception des femmes, et que le documentaire cherche à mettre en évidence.

Utilisée en début de roman, la photo de l'actrice permet aussi d'installer le lien thématique entre le documentaire et l'intrigue principale, par un jeu de contraste humoristique qui oppose symboliquement deux femmes: Susana Estrada, l'icône fantasmée d'un univers irréel, et Sonia Griñán, la mère de famille ancrée dans son quotidien domestique. Dans le chapitre qui suit la caja 1, on découvre ainsi les réactions de Sonia, affairée dans la cuisine, à la publication de la photo dans la presse : "Qué guarra, la tía [...] ¿No le dará vergüenza? [...] Pero ¿cómo puede atreverse una mujer a hacer estas cosas?» (Sanz, 2013 : 22-23). Paradoxalement, le dévoilement public du corps féminin censé incarner une forme de libération ne semble pas convaincre les principales intéressées... L'opposition initiale entre ces deux personnages de femmes, a priori interprétable comme un conflit de valeurs entre modernité et conservatisme, s'avère dans la suite du roman bien plus subtile qu'il n'y paraît : les évènements narrés démontreront à la jeune narratrice et au lecteur que la plus avant-gardiste des deux n'est pas l'actrice qui montre son sein, mais la mère de famille provinciale de classe moyenne qui osera braver les lois et les tabous de son époque pour affirmer la liberté de son choix : celui d'avorter.

Le propos du documentaire fictif se dévoile progressivement au fil des cajas successives, depuis la photo de Susana Estrada en 1978, image d'une provocation encore maîtrisée, audacieuse, non dépourvue peut-être d'un certain charme, jusqu'à la télévision des années 2000 où les mêmes figures devenues pathétiques se donnent en spectacle pour 
prolonger autant que possible leur existence médiatique, au risque d'y perdre leur propre dignité. Les nombreuses actrices évoquées le sont essentiellement par la déchéance survenue après une période de gloire éphémère, ce qui donne même lieu à une énumération macabre qui occupe une page entière du roman: "la colección de muertes prematuras y destinos rotos que pueblan las páginas de un voluminoso cronicón amarillo " (Sanz, 2013: 114). La liste n'est pas seulement écrite, sous la forme d'une seule phrase interminable, mais aussi lue à voix haute par la voix off, et surtout soulignée par une succession d'images « vertiginosas y psicodélicas » (Sanz, $2013: 113)$ qui renforce par un procédé visuel l'effet d'étourdissement produit par la succession lancinante des noms d'actrices. Dans quel but? Forcer les (télé)spectateurs à ouvrir les yeux sur l'envers du décor, jusqu'à l'écœurement ? Pas seulement.

16 La démarche de la réalisatrice correspond à une forme d'engagement qui ne dit pas explicitement son nom, qui n'utilise pas la voie pamphlétaire ni politique, mais qui présente des faits, une réalité, en les accompagnant d'une analyse à plusieurs dimensions. La réflexion sur le statut de la femme-objet, de la femme-jouet, est ainsi omniprésente dans le documentaire et débouche sur une interprétation sociologique aboutie du phénomène du destape, toujours en lien avec l'image et le regard :

El desnudo se transforma en destape cuando se vacía de sentido y de oportunidad, y sobre todo cuando se enfoca en primer plano la mirada del macho y el movimiento: la represión sexual, el morderse los labios y sacar los ojos de las órbitas ante la contemplación de un cuerpo de mujer, cada acción inhibida se desata, [...]. (138-139)

17 Le droit à la nudité, à l'exhibition, au dévoilement, synonyme d'un certain accès à la liberté après quarante années de dictature, n'est en fait que prolongation de la domination masculine et soumission à un autre pouvoir totalitaire, celui du profit économique: "Las mujeres disponen libremente de las partes comerciales de su anatomía, aunque el comercio sea tal vez incompatible con la libertad. El paso que dan hacia delante es casi siempre irreversible.» (Sanz, $2013: 188)$; ce dernier extrait prend place dans la caja 8, " Subasta", consacrée à un internaute acheteur compulsif d'anciennes couvertures du magazine Interviú qui mettent en scène les actrices du destape. Le documentaire ne se contente pas de démythifier les héroïnes de papier glacé qui font rêver Catalina jeune fille, mais porte également un regard acéré sur un phénomène culturel et moral trop vite assimilé à une forme de progrès dans un contexte historique particulier, celui de la transition démocratique. ${ }^{4}$

Daniela Astor y la caja negra met en relation le destape avec une évolution de société capitale dans la vie des femmes: le droit à l'avortement, qui questionne également, sous une autre forme, le droit à disposer de son propre corps. Le traitement du thème passe par le remarquable jeu d'échos, le réseau de sens, la discrète interaction qui se tisse tout au long du roman entre les différents médias et niveaux de récits. La grossesse de Sonia apparaît de façon progressive dans le principal fil narratif à la première personne, à travers le regard de Catalina enfant qui découvre peu à peu le secret que ses parents lui cachent. Puis la caja 7, "Españolas en París ", analyse la représentation de l'avortement dans la filmographie de la transition démocratique, avant de faire un point d'information sociologique et juridique sur le sujet. Le documentaire audiovisuel fictif, qui porte lui-même sur le cinéma, illustre par l'image et le commentaire une question de société fictionnalisée dans l'intrigue du roman par le point de vue limité d'une enfant. C'est seulement dans le dernier chapitre du récit à la première personne que la boucle se ferme avec une grande subtilité pour souligner encore une fois le contraste entre l'image véhiculée par certains médias et la réalité : le 
texte consiste en une succession de phrases négatives qui nient l'existence réelle de tous les clichés misérabilistes et les stéréotypes culpabilisants utilisés par les films de l'époque ; un anti-récit de la scène de l'avortement:

Mi madre no abortó en una mesa de cocina con las piernas colgando.

La persona que le practicó el aborto no fue una remendadora de virgos ni una santera que

llenó la habitación de pestíferos vapores desinfectantes y humo de veguero.[...]

A mi madre no la anestesiaron con éter ni perdió la conciencia. [...]

Mi madre no vio cuchillos con el filo rojo de óxido. Ni trapos. Ni jeringuillas sin hervir

cargadas de un líquido blancuzco. No la sujetaron con correas a una camilla con las sábanas

salpicadas de secreciones de la anterior paciente. [...]

Nada sucedió así. Mi madre me ha jurado que nada de eso sucedió. (Sanz, 2013 : 237-238)

La longue litanie des négations vient déconstruire, phrase après phrase, un discours monolithique et totalitaire, aux accents de propagande, et permet le rétablissement d'une vérité paradoxale: en 1978, malgré un code pénal qui sanctionne encore les femmes et les médecins, il est possible en Espagne d'avoir recours à l'avortement dans des conditions humaines et sanitaires décentes, en clinique privée, dans la clandestinité, ce qui constitue malgré tout un progrès plus important pour les femmes espagnoles que le destape, un premier pas un peu plus ferme vers la liberté, une petite avancée obtenue dans la souffrance par des femmes qui ressemblent davantage à Sonia Griñán qu'à Susana Estrada ${ }^{5}$. La technique narrative utilisée par Marta Sanz est indirecte mais brutale, les descriptions sont crues, sèches, sans artifice. Si la séquence racontée n'est pas celle qu'a vécue le personnage, elle n'en est pas moins réaliste et sans doute bien réelle, mais à une époque et dans un milieu différents; elle est aussi d'un autre âge, tout comme le procès de Sonia, brièvement raconté dans la suite du récit : le fonctionnement de l'institution judiciaire, encore profondément dominé par les valeurs du franquisme, est un autre révélateur des lenteurs et des paradoxes de la transition démocratique.

\section{Regards et voix}

Si le documentaire et le récit à la première personne interagissent aussi bien dans Daniela Astor y la caja negra, c'est en grande partie parce qu'ils sont assumés par la même auteure, malgré les trente-cinq ou quarante ans qui séparent les deux moments d'énonciation. Entre le point de vue de l'enfant et celui de l'adulte, il existe bien entendu de nombreuses différences qui se justifient notamment par l'une des clés du roman : la perte de l'innocence, symbolisée par la disparition progressive de la figure imaginaire de Daniela Astor. Mais le regard porté sur le monde par Catalina l'année de ses douze ans est déjà celui d'une jeune fille avide de comprendre et d'explorer le monde (des adultes): sa précocité, sa curiosité, sa détermination à recueillir des informations, sa capacité de déduction et d'analyse subjective, son sens de la psychologie humaine, sont autant d'atouts pour une future documentariste, et prennent progressivement le pas sur son goût plus infantile pour l'imaginaire. Ce sera d'ailleurs pour elle l'occasion de découvrir que l'accès à la connaissance et à la compréhension mène à une forme de lucidité qui peut être synonyme de souffrance : " Me enteraré de todo, como siempre antes de tiempo, pero lo haré con mucha discreción. Después me dolerá el estómago y no podré dormir » (Sanz, 2013 :153). Dans sa trajectoire vers la maturité, Catalina perdra toutes ses illusions sur le monde des actrices qui la faisaient 
tant rêver ; au point de devenir, plus tard, une démystificatrice accomplie sur ce même sujet.

Il faut en réalité attendre la moitié du roman pour avoir l'assurance que la réalisatrice du documentaire, pourtant identifiée comme Catalina H. Griñán dès le début de la caja 1 page 17, est également son interprète dans le rôle de l'intervieweuse meneuse d'enquête, ainsi que l'auteure et probable énonciatrice du commentaire oral via la voix off. Marta Sanz semble différer volontairement cette explicitation en préservant l'anonymat en début de roman, peut-être pour ne pas brouiller la perception que l'on a de l'enfant narratrice, dont le point de vue est dans un premier temps dominant: "Se oye la voz de la persona que ha ido a entrevistar a Leguna» (Sanz, 2013 : 38), mentionne l'ekphrasis du documentaire dans les portions en caractères gras. C'est seulement page 138 que la réalisatrice fait irruption comme par effraction dans son propre documentaire, à la fin de la caja 6, «Destape »:

Corte: Catalina H. Griñán mira a cámara. Es una mujer de casi cincuenta años que parece un poco más joven. Menuda. [...] Catalina, sin maquillar, mira a cámara y se burla del texto en off. Lo remeda malintencionadamente: "Una guapísima Patricia Adriani », " Nadiuska hace una exhibición de lencería", "Valerio Lazarov, verdadero espeleólogo de talentos femeninos». Catalina hace una pausa. Se ríe : ¿Quién está hablando por mi boca?» [...] Catalina guiña un ojo a cámara: "Por supuesto esto no pretende ser un mensaje subliminal, sino una clarísima injerencia de autor ». (Sanz, $2013: 138$ )

Les retouches de texte évoquées correspondent à ce que le lecteur a pu lire quelques lignes plus haut, elles ont donc déjà été effectuées, et affirment le pouvoir de Catalina en tant qu'auteure. Dans les trois cas il s'agit d'extraits où la subjectivité de l'énonciatrice est évidente, soit par l'emploi d'un adjectif évaluatif à la forme superlative ("guapisima»), soit par l'utilisation de formulations ironiques ("una exhibición de lencería », "espeleólogo de talentos fememinos »). Son intervention ex abrupto dans le fil du documentaire semble vouloir attirer notre attention sur le procédé, comme pour souligner que l'objectivité est impossible, et sans doute même inopportune ; c'est d'ailleurs d'après Mauro ce qui distingue la démarche documentaire de celle du journaliste, à savoir l'opposition entre «la prétention à l'“objectivité" des programmes audiovisuels relevant du journalisme » et la "subjectivité assumée, et explicitée, lisible pour le spectateur » du documentariste (Mauro, $2005: 19$ ).

Pour bien apprécier ces notions lorsqu'elles s'appliquent à un documentaire, il convient de prendre en compte que celui-ci se compose de matériaux très divers, dont le contenu n'a pas forcément été écrit par l'auteure en amont de la réalisation, notamment les actions et paroles a priori spontanées des protagonistes, saisies sur le vif par les caméras ou les micros. La question de l'énonciation dans le documentaire s'avère ainsi particulièrement complexe, jusqu'à s'effacer peut-être derrière le poids de la réalité :

Apparaît donc ici, dans les faits, une forme de réalité-auteur. Celle-ci est forcément dérangeante, puisqu'elle relève d'une sociologie de cet art et que comme l'analyse Pierre Bourdieu : « La sociologie et l'art ne font pas bon ménage. Cela tient à l'art et aux artistes qui supportent mal tout ce qui attente à l'idée qu'ils ont d'eux-mêmes: l'univers de l'art est un univers de croyance, croyance dans le don, dans l'unicité du créateur incréé, et l'irruption du sociologue qui veut comprendre, expliquer, rendre raison, fait scandale » (Bourdieu : 1984) ${ }^{6}$. Donc, précisément, scandale il y a et, dans le documentaire, la notion d'auteur ne soit surtout pas être "réifiée ", car la création reste redevable au vécu de personnes qui n'ont souvent aucun rapport avec les industries de programmes audiovisuels, et qui ne retirent généralement 
aucun dividende ni financier, ni moral, ni de notoriété de l'œuvre qui leur a été consacrée. (Mauro, 2005 : 29) nous intéresse, en particulier grâce aux différents niveaux de mise en abyme : puisqu'il porte sur le cinéma, il inclut bien évidemment des séquences filmiques, mais aussi des extraits d'archive télévisuels, des images tirées d'internet, et de façon plus classique des interviews. L'ordonnancement de cette diversité, du point de vue de l'analyse littéraire, relèverait de ce que l'on appelle la régie narrative, dont la conception se rapproche assez de la notion de "réalité-auteur", mais préserve malgré tout l'existence d'un architecte, d'un régisseur, d'un monteur en termes audiovisuels, qui donne et distribue la parole, agence les différents éléments, et assume la responsabilité du produit final, y compris dans la part de manipulation qu'il peut comporter et qui apparait très souvent lorsque l'on choisit de mélanger des ingrédients réels et fictionnels. Preuve en est le recours au procédé de la reconstitution ${ }^{7}$, appelée " dramatización filmada " lorsqu'il s'agit de l'assassinat de Sandra Mozarowsky (Sanz, 2013 : 113) ou "simulación» pour la rencontre entre Rafael Reig et Amparo Muñoz (Sanz, 2013: 80) : les personnes objets du documentaire sont alors remplacées par des comédiens, parfois filmés de loin ou de dos pour mieux faire illusion et limiter l'effet de fiction. La complexité du rapport au réel s'accentue encore si l'on considère que certaines des protagonistes du documentaire, auxquelles la création est redevable selon Mauro, sont elles-mêmes des actrices en quête de notoriété, dont la sincérité est à relativiser tant elles sont prisonnières de leur désir d'exhibition.

Le documentaire réalisé par Catalina H. Griñán est intégré de façon subtile et efficace, via un réseau thématique, à l'intrigue du roman, mais il n'en demeure pas moins une entité à part entière, au statut indéterminable. Comment interpréter en effet les scènes d'interviews de personnages réels, comme celle du réalisateur Javier Maqua et de sa compagne actrice Gloria Berrocal, qui constitue l'essentiel de la caja 9? S'agit-il de l'ekphrasis d'un entretien qui aurait vraiment eu lieu ${ }^{8}$ ? Mais mené par qui, puisque Catalina est bel et bien, elle, un personnage de fiction? La liste des références électroniques proposée par Marta Sanz à la fin du roman n'est pas d'un très grand secours, contrairement à ce que pourrait laisser croire la présentation qu'en fait l'auteure :

Todos los blogs, vídeos y páginas web que se citan en esta novela están ubicados realmente en algún lugar de la red. Ofrecemos sus direcciones electrónicas por si al lector curioso le apetece visitarlos y porque la Historia y la Ficción no son lo mismo, por mucho que se interrelacionen. (Sanz, $2013: 265$ )

Impossible malgré cela de trouver là les références de tous les documents cités ou évoqués, sauf à considérer que ce manque d'exhaustivité est précisément la clef d'interprétation des différents éléments composant le documentaire : ceux qui ne sont pas listés sont peut-être bien ceux qui sont fictionnels, à l'instar de l'interview de Javier Maqua et Gloria Becorral, ou encore de l'émission de télévision, "un programa del corazón » (Sanz, 2013 : 243), qui occupe la caja 10 et les vingt dernières pages du roman.

arta Sanz nous offre avec Daniela Astor y la caja negra un formidable portrait de jeune fille pendant la transition démocratique, mais aussi une analyse acérée du phénomène du destape et du destin des femmes pendant cette période. La forme hybride du roman, qui fait cohabiter le récit de l'enfant à la première personne avec un documentaire audiovisuel fictif, crée un pacte de lecture ambigu mais redoutablement efficace, un peu à la façon de Soldados de Salamina (2001) de Javier Cercas, qui a donné lieu à tant de 
travaux, de commentaires et de polémiques à propos de cette audacieuse porosité entre réalité et fiction. Pour Marta Sanz, la réception de la même stratégie narrative est très différente, car le mélange des genres ne s'étend pas à l'axiologie ni à l'idéologie, qui se dégage toujours de ses œuvres de manière implicite, fermement mais discrètement, avec une extrême subtilité. Le faux documentaire ne permet pas seulement de poser la problématique du lien entre réalité et fiction, mais aussi de construire une réflexion quasi performative (le roman fait ce qu'il dit) sur la représentation de la réalité, que ce soit par la photo, la télévision, le cinéma ou internet. Tout cela sans jamais perdre le lien avec l'intrigue romanesque et son magnifique personnage de femme en devenir, dans un roman résolument et intrinsèquement féminin.

\section{BIBLIOGRAPHIE}

BESSON Rémy (2014), « Prolégomènes pour une définition de l'intermédialité », en ligne sur le portail HAL de l'Université Toulouse - Jean Jaurès : <http://hal-univ-tlse2.archives-ouvertes.fr/ hal-01012325 (24 juillet 2018).

FERNÁNDEZ CUESTA Manuel (1997), « Marta Sanz. La libertad y la palabra », en ligne sur Mundo obrero : <http://www.eurosur.org/mo.old/0498/0498culturamartasanz.htm> (19 juillet 2018).

MAURo Didier (2005), Le documentaire, Paris, France : Dixit.

SANZ Marta (2013), Daniela Astor y la caja negra, Madrid, Espagne : Anagrama.

\section{NOTES}

1. Pour une hybridation complète, incluant une dimension matérielle concrète, il faut imaginer la possibilité d'associer plus intimement les deux médias (texte écrit et vidéo) : on peut l'envisager aisément pour la lecture numérique, grâce en particulier aux liens hypertextes, mais dans le cas du support papier traditionnel, le roman devrait être produit et commercialisé en étant accompagné d'un DVD interactif à découvrir de façon simultanée, ce qui modifierait considérablement la nature même de l'acte de lecture, et relègue de fait ce type de proposition au rang de l'expérimentation artistique. Le média écrit ne peut intégrer concrètement le document audiovisuel que sous la forme de sa transcription écrite préalable à la réalisation (script ou scénario) ou postérieure à la diffusion (description du document d'un point de vue spectateur). On touche là, sans doute, aux limites de l'intermédialité pour le texte littéraire, tout du moins sous sa forme imprimée, qui restreint ses capacités d'accueil aux mots et aux images reproductibles sur papier. Le champ des hybridations possibles est a priori beaucoup plus large pour d'autres médias artistiques, comme le théatre par exemple, qui peut accueillir sans les dénaturer et sans se trahir des documents de toutes natures: sonores, visuels, voire plurisensoriels et interactifs.

2. En France, ce rôle de conservation et de sauvegarde du fonds est celui de l'Institut National de l'Audiovisuel (INA), créé en 1974. En Espagne, de nombreuses archives sont accessibles grâce au groupe audiovisuel public Radio Televisión Española (RTVE). 
3. Susana Estrada, María José Cantudo, Amparo Muñoz ou Bárbara Rey.

4. La transition démocratique espagnole, longtemps idéalisée dans sa globalité, est aujourd'hui l'objet d'une analyse beaucoup plus critique portée par les historiens, par les acteurs de l'époque et par les romanciers. On peut considérer que le texte de Marta Sanz s'inscrit dans le même type de démarche, en dénonçant les effets pervers d'une libération des mœurs parfois précipitée, mal digérée, ou récupérée et dévoyée par les protagonistes de la dictature eux-mêmes. Le phénomène $\mathrm{du}$ destape n'est qu'une vitrine de liberté, comme dans une certaine mesure celui de la Movida, que Marta Sanz analyse ainsi dans une interview accordée à Manuel Fernández Cuesta en 1997 pour Mundo Obrero: "Y aunque Tierno Galván fuera un señor muy respetable en otros aspectos, lo que hizo en Madrid y en otros lugares con la famosa « Movida » fue recurrir al tema de « Pan y Toros ». Vamos a liberarnos, hagamos lo que no hemos hecho durante mucho tiempo y salgamos a la calle no a protestar, sino a reírnos, divertirnos y para demostrarle a todo el mundo lo libres y estupendos que somos. En este sentido por supuesto que el PSOE tiene mucha responsabilidad al crear un clima de libertad ficticia que además degradó la cultura.» (http://www.eurosur.org/mo.old/0498/0498culturamartasanz.htm, page consultée le 19/07/2018). Le règne unanime du divertissement roi, qui joue le rôle de défouloir ou d'exutoire après les années de dictature national-catholique, occulte la pauvreté de certaines des manifestations culturelles proposées et la perte de conscience politique qui les accompagne.

5. Ces deux modèles féminins ne sont peut-être pas complètement incompatibles, comme semble le suggérer la référence à Ana Belén à la fin de la caja 7 , consacrée au thème de l'avortement. L'actrice, l'une des premières à avoir dévoilé sa poitrine sur un écran de cinéma espagnol dans le film El amor del capitán Brando en 1974, interprétait en 1971 dans Españolas en París une scène d'avortement contraint, sur une table de cuisine, par deux vieilles femmes sordides aux allures de sorcières. La fin de la caja 7 souligne, dans son dernier paragraphe, qu'Ana Belén fera partie quelques années plus tard, en 1979, des 1300 femmes signataires d'un document public où elles déclaraient : « Yo he abortado voluntariamente ».

6. La référence utilisée par Didier Mauro est la suivante: BouRDIEU Pierre, « Mais qui a créé les créateurs? », P. Bourdieu, Questions de sociologie, Paris : Minuit, 1981, p. 207.

7. La reconstitution est au cœur de ce que l'on appelle le docudrame (docudrama en anglais), qui consiste à fictionnaliser et dramatiser des évènements réels sous la forme d'un documentaire. À ne pas confondre avec le docufiction, qui lui est un véritable documentaire utilisant des outils propres à la fiction.

8. C'est ce que semble suggérer la notice de l'encyclopédie en ligne Wikipedia consacrée à Gloria Berrocal (https://es.wikipedia.org/wiki/Gloria_Berrocal, page consultée le 19/07/2018), en indiquant en note : "En una entrevista filmada con Gloria y Maqua, y recogida por Marta Sanz en su libro Daniela Astor y la caja negra (ver: Referencias); el propio Maqua relata la anécdota ocurrida durante una de las reuniones de los "Once" con las autoridades y personalidades del último gobierno de Francisco Franco [...] ». Le roman de Marta Sanz est ainsi considéré dans sa globalité comme un documentaire qui fait foi, et qui dispense même l'auteur de la notice de renvoyer le lecteur vers ce qui serait la véritable référence de cette interview. Le manque de rigueur dans la citation des sources, sans compter la confusion entre le personnage du roman et son auteure, ne plaide évidemment pas en la faveur de Wikipedia quant à sa fiabilité. 


\section{RÉSUMÉS}

Le roman de Marta Sanz, Daniela Astor y la caja negra, paru en 2013, propose une forme d'intermédialité littéraire très originale en faisant alterner le récit avec le script d'un documentaire audiovisuel fictif dont le lien subtil avec l'intrigue principale génère de profonds effets de sens. La réalisatrice du documentaire, qui n'est autre que la jeune narratrice parvenue à l'âge adulte, retrace ainsi le destin pathétique des actrices du destape qui la faisaient tant rêver à l'âge de douze ans, pendant la transition démocratique espagnole. L'étude des procédés narratifs mis en œuvre et de leurs effets révèle l'existence d'une stratégie d'hybridation médiatique novatrice, au service de la démythification des icônes d'une époque charnière, aussi bien pour le pays que pour la jeune protagoniste.

Marta Sanz's novel, Daniela Astor y la caja negra, published in 2013, relies on a highly original form of literary intermediality. The story alternates with the script of a fictional audio-visual documentary whose relationship with the main plot is the key to meaning. The director of the documentary, who is in fact none other than the young narrator grown into an adult, relates the pathetic destiny of the destape actresses whom she used to dream about during the Spanish democratic transition. The study of the narrative devices used and of their effects reveals the existence of a new strategy of media hybridization, which serves to debunk the icons of a crucial era both for the country and for the young protagonist.

\section{INDEX}

Keywords : contemporary Spanish novel, Marta Sanz, intermediality, documentary, Spanish democratic transition, destape

Mots-clés : roman espagnol contemporain, Marta Sanz, intermédialité, documentaire, transition démocratique espagnole, destape

\section{AUTEUR}

\section{MYRIAM ROCHE}

LLSETI, Université Savoie Mont Blanc 